\title{
DATA ENVELOPMENT ANALYSIS WITH FUZZY COMPLEX NUMBERS WITH AN EMPIRICAL CASE ON POWER PLANTS OF IRAN
}

\author{
MAHMOOD Esfandiari ${ }^{1}$ AND SABer SAATI ${ }^{2, *}$
}

\begin{abstract}
Using Data Envelopment Analysis (DEA) in complex environment is an idea that has recently presented for measuring the relative efficiencies of a set of Decision Making Units (DMUs) with complex inputs and outputs. The values of the input and output data in real-world problems appear sometimes as fuzzy complex number. For dealing with these types of data in DEA, we need to design a new model. This paper proposes a DEA model with triangular fuzzy complex numbers and solve it by using the concept of the data size and the $\alpha$-level approach. This method transforms DEA model with fuzzy complex data to a linear programing problem with crisp data. In the following, a ranking model is also developed using the above approach to rank the efficient DMUs. The proposed method is presented for the first time by the authors and there is no similar method. Finally, we present a case study in the generators of the steam power plants to demonstrate the applicability of the proposed methods in the power industry.
\end{abstract}

Mathematics Subject Classification. 90C08, 90C70, 97F50.

Received March 18, 2020. Accepted June 22, 2020.

\section{INTRODUCTION}

Data Envelopment Analysis (DEA) was originally derived from Farrell [9] model and was presented by Charnes et al. [6], is a set of non-parametric Linear Programming (LP) techniques for evaluating the relative efficiency of a group of homogeneous Decision Making Units (DMUs) with multiple inputs and outputs. This model is known as CCR model. Conventional DEA is sensitive to input and output data of DMUs and when inputs and outputs are imprecise, it is difficult to calculate the efficiency of these units. Therefore, since 1978, a lot of studies has been done in field of DEA in terms of various types of data (precise and imprecise data). Although many researches has been done in the field of DEA, but there are still some challenging issues associated with DEA. One of the challenging issues is that the input and output parameters of DMUs became as fuzzy complex, in which case in order to calculate the efficiency of these DMUs, it is practically impossible to use existing DEA methods to handle this type of data. Each Fuzzy Complex Number (FCN) consists of two real and imaginary parts where both parts are available as Fuzzy Numbers (FNs) [4]. Some of the application of this type of data can be mentioned in power plant, mechanic and telecommunication industries.

Keywords. Data Envelopment Analysis, fuzzy number, complex number, ranking, power plant.

1 Department of Industrial Engineering, Faculty of Industrial Engineering, South Tehran Branch, Islamic Azad University, Tehran, Iran

2 Department of Mathematics, North Tehran Branch, Islamic Azad University, Tehran, Iran.

* Corresponding author: s_saatim@iau-tnb.ac.ir 
We briefly review Fuzzy DEA (FDEA) models in the literature over the past years. For the first time, the FDEA model was presented by Sengupta [17] in which the tolerance approach was used to incorporate uncertainty. The possibility approach, one of the used methods to deal with fuzzy data in DEA, was initially extended by Guo et al. [11]. Guo and Tanaka [10] initially proposed a FDEA model using the fuzzy ranking approach in which fuzzy constraints were transformed to crisp constraints by predetermining possibility level. Lertworasirikul et al. $[13,14]$ presented two approaches of the possibility and the credibility to solve the ranking problem in FDEA models. Saati et al. [16] introduced a fuzzy DEA model with asymmetrical triangular FNs using the $\alpha$-level based approach. They transformed the fuzzy CCR model into a crisp LP problem that could be solved for different values of $\alpha$. Hatami-Marbini et al. [12] classified the existing approaches to dealing with fuzzy data in DEA into four categories: the $\alpha$-level based approach, the tolerance approach, the possibility approach and the fuzzy ranking approach. Azadi et al. [2] presented an integrated FDEA model in order to assess the performance of suppliers in sustainable supply chain management with the aim of selecting the best sustainable supplier. Tlig and Hamed [19] evaluated the efficiency of commercial Tunisian Banks by using FDEA model based on the possibility approach and the approach based on relations between FNs. Bai et al. [3] developed a FDEA model with parametric interval-valued fuzzy variable-based inputs and outputs was modeled as fuzzy expectation model with credibility constraints. Mirzaei and Salehi [15] extended a new model to calculate ranking efficient DMUs in the fuzzy environment. Their idea for developing this model was to use the Tchebycheff norm with fuzzy data in DEA. Tavassoli et al. [18] introduced a novel stochastic-fuzzy DEA model using $\alpha$-level method for evaluating supplier's sustainability in supply chain management.

In field of DEA with complex data, a paper is published. For the first time, DEA with complex numbers was introduced by Esfandiari et al. [8] and it was applied in a case study of the power plant generators in Iran. They transformed a DEA model with complex numbers into a LP problem in real environment by using the concept of the size of the linear combination of inputs and outputs of all units in constraints of model. This model requires accurate measurement of both the parts of the real and imaginary of complex inputs and outputs, while the observed values of these two parts in real-world problems are often fuzzy or imprecise, which may lead to inaccurate results.

As seen in the literature review, although a lot of research has been done in the field of FDEA, there is no DEA model with the purpose of measuring the efficiency of DMUs with fuzzy complex number that simultaneously deals with complexity and fuzziness of the data and this method is presented for the first time. Hence, we did not bring any similar articles for review. According to the existing technical gap in the field of DEA models, we propose a new DEA model with fuzzy complex data and its ranking model by using the proposed modified method and the $\alpha$-level approach proposed by Saati et al. [16].

The rest of the paper is organized as follows: In Section 2, the required DEA models are reviewed. In Section 3, a new DEA model and its ranking model are extended for FCNs. Section 4 provides a case study from the generators of the power plants to show how the models presented in Section 3 can be used. Conclusion and future research directions are presented in Section 5 .

\section{Data envelopment analysis and Related teChniques}

\subsection{CCR model}

DEA is a nonparametric technique for evaluating the relative efficiency of a set of DMUs with multiple input-output [6]. Suppose we want to analyses the efficiency of $n$ DMUs which each DMU consumes $m$ inputs to product $s$ outputs. In the model, $x_{i j}(i=1, \ldots, m)$ and $y_{r j}(r=1, \ldots, s)$ are the value of the $i$ th input and the $r$ th output of $j$ th DMU $(j=1, \ldots, n)$, respectively that all these parameters are non-negative. Primal and dual programing of CCR models for $\mathrm{DMU}_{p}$ are defined as follows: 


$$
\begin{array}{ll}
\text { Max } & z=\sum_{r=1}^{s} u_{r} y_{r p}, \\
\text { s.t. } & \sum_{i=1}^{m} v_{i} x_{i p}=1, \\
& \sum_{r=1}^{s} u_{r} y_{r j}-\sum_{i=1}^{m} v_{i} x_{i j} \leq 0, \quad j=1, \ldots, n \\
& u_{r}, v_{i} \geq 0, \\
& r=1, \ldots, s ; \quad i=1, \ldots, m
\end{array}
$$

$\operatorname{Min} z=\theta$

$$
\begin{aligned}
& \text { s.t. } \sum_{j=1}^{n} \lambda_{j} x_{i j} \leq \theta x_{i p}, \quad i=1, \ldots, m \\
& \sum_{j=1}^{n} \lambda_{j} y_{r j} \geq y_{r p}, \quad r=1, \ldots, s \\
& \lambda_{j} \geq 0, \quad j=1, \ldots, n .
\end{aligned}
$$

The LP models (2.1) and (2.2) are called the multiplier and envelopment models of CCR, respectively under constant returns to scale (CRS) assumption.

\subsection{DEA with complex numbers}

If the input and output parameters of DMUs appear as complex numbers and the purpose is to calculate the efficiency these DMUs, conventional DEA models cannot deal with this case. Therefore, CCR model with complex numbers was designed by Esfandiari et al. [8]. In model (2.1), consider $x_{i j}=x_{i j}^{a}+\mathrm{i} x_{i j}^{b}, i=1, \ldots, m$ as complex inputs and $y_{r j}=y_{r j}^{a}+\mathrm{i} y_{r j}^{b}, r=1, \ldots, s$ as complex outputs where $a$ and $b$ are symbols to identify the real and imaginary parts of the parameters, respectively. The CCR model with incorporating complex data by applying the concept of the size of the linear combination of complex data in constraints is as follows [8]:

$$
\begin{aligned}
& \operatorname{Min} z=\theta \\
& \text { s.t. } \sum_{j=1}^{n} \lambda_{j j}\left(\left(x_{i j}^{a}\right)^{2}+\left(x_{i j}^{b}\right)^{2}\right)+2 \sum_{j=1}^{n-1} \sum_{h=j+1}^{n} \lambda_{j h}\left(x_{i j}^{a} x_{i h}^{a}+x_{i j}^{b} x_{i h}^{b}\right) \leq \theta\left(\left(x_{i p}^{a}\right)^{2}+\left(x_{i p}^{b}\right)^{2}\right), \quad i=1, \ldots, m \\
& \sum_{j=1}^{n} \lambda_{j j}\left(\left(y_{r j}^{a}\right)^{2}+\left(y_{r j}^{b}\right)^{2}\right)+2 \sum_{j=1}^{n-1} \sum_{h=j+1}^{n} \lambda_{j h}\left(y_{r j}^{a} y_{r h}^{a}+y_{r j}^{b} y_{r h}^{b}\right) \geq\left(\left(y_{r p}^{a}\right)^{2}+\left(y_{r p}^{b}\right)^{2}\right), \quad r=1, \ldots, s \\
& \lambda_{j j}, \lambda_{j h} \geq 0, \quad j=1, \ldots, n \text {; } \\
& h=2, \ldots, n ; \quad \theta \text { free. }
\end{aligned}
$$

Model (2.3) is a LP problem, which evaluate the performance of DMUs with complex data. This model meets the requirement of the efficiency of DEA model and always gives the performance values between 0 and 1 . However, this model has some weaknesses: (A) This model has a complicate structure due to the use of the size of the linear combinations of data, (B) If we need a variable value of $\lambda_{j}$, we cannot obtain the value of $\lambda_{j}$ from the value of $\lambda_{j j}$ or $\lambda_{j h}$.

\subsection{Fuzzy DEA}

In the classical DEA models, the input and output data are expressed as precise, whereas in many applications the data are imprecise or fuzzy and the traditional DEA models cannot deal with them. Suppose all of the inputs and outputs in model (2.1) are represented as the triangular fuzzy numbers. Consider the fuzzy inputs as $\left(x_{i j}^{(l)}, x_{i j}^{(m)}, x_{i j}^{(u)}\right)$ and the fuzzy outputs as $\left(y_{r j}^{(l)}, y_{r j}^{(m)}, y_{r j}^{(u)}\right)$. In this case, the proposed model of DEA with the fuzzy numbers by using the $\alpha$-level based approach and its ranking model are given as bellows [16]: 


$$
\begin{aligned}
& \operatorname{Max} z=\sum_{r=1}^{s} \bar{y}_{r p} \\
& \text { s.t. } \sum_{i=1}^{m} \bar{x}_{i p}=1 \text {, } \\
& \sum_{r=1}^{s} \bar{y}_{r j}-\sum_{i=1}^{m} \bar{x}_{i j} \leq 0, \quad \forall j \\
& v_{i}\left(\alpha x_{i j}^{(m)}+(1-\alpha) x_{i j}^{(l)}\right) \leq \bar{x}_{i j} \leq v_{i}\left(\alpha x_{i j}^{(m)}+(1-\alpha) x_{i j}^{(u)}\right), \quad \forall i, j \\
& u_{i}\left(\alpha y_{r j}^{(m)}+(1-\alpha) y_{r j}^{(l)}\right) \leq \bar{y}_{r j} \leq u_{i}\left(\alpha y_{r j}^{(m)}+(1-\alpha) y_{r j}^{(u)}\right), \quad \forall r, j \\
& u_{r}, v_{i} \geq 0, \quad \forall r, i .
\end{aligned}
$$

$\operatorname{Min} z=\theta$

$$
\begin{aligned}
& \text { s.t. } \sum_{j=1}^{n} \lambda_{j}\left(\alpha x_{i j}^{(m)}+(1-\alpha) x_{i j}^{(u)}\right) \leq \theta\left(\alpha x_{i p}^{(m)}+(1-\alpha) x_{i p}^{(l)}\right), \quad \forall i \\
& \sum_{j=1}^{n} \lambda_{j}\left(\alpha x_{i j}^{(m)}+(1-\alpha) y_{r j}^{(l)}\right) \geq \alpha y_{r p}^{(m)}+(1-\alpha) y_{r p}^{(u)}, \quad \forall r
\end{aligned}
$$

$\bar{x}_{i p}$ and $\bar{y}_{r p}$ are the bounded variables whose upper and lower bounds are specified in the third and fourth sets of constraints, respectively. $\alpha$ is a specified parameter between 0 and 1 . In fact, a fuzzy CCR model was suggested as possibilistic programing problem and converted into an interval programing problem which like the crisp LP model can calculates the efficiency of DMUs with the fuzzy data for any different value of $\alpha$ parameter. Saati et al. [16] also developed a ranking model to rank efficient DMUs that used the envelopment CCR model by (2.2) and the $\alpha$-level based approach for developing the new model. Model (2.2) by considering the fuzzy inputs and outputs was transformed into the crisp parametric LP problem can rank efficient DMUs for any amount of $\alpha$.

\section{Proposed Models}

\subsection{Proposed modified method for Esfandiari et al. [8]}

In this section, we present a modified model of Esfandiari et al. [8]. Instead of using the size of the linear combination of the complex data of all DMUs in CCR model, we use the size of the complex data of all DMUs as follows:

$$
\begin{aligned}
& \operatorname{Min} z=\theta \\
& \text { s.t. } \sum_{j=1}^{n} \lambda_{j}\left|\left(x_{i j}^{a}+\mathrm{i} x_{i j}^{b}\right)\right| \leq \theta\left|\left(x_{i p}^{a}+\mathrm{i} x_{i p}^{b}\right)\right|, \quad i=1, \ldots, m \\
& \sum_{j=1}^{n} \lambda_{j}\left|\left(y_{r j}^{a}+\mathrm{i} y_{r j}^{b}\right)\right| \geq\left|y_{r p}^{a}+\mathrm{i} y_{r p}^{b}\right|, \quad r=1, \ldots, s \\
& \lambda_{j} \geq 0, \quad j=1, \ldots, n ; \quad \theta \text { free. }
\end{aligned}
$$

According to the properties of complex numbers [7], the size of a complex number is equal to the second root of the sum of the squares of the real and imaginary parts. We use the second power of this size for simplicity in calculations and will have:

$$
\begin{aligned}
& \text { Min } z=\theta \\
& \text { s.t. } \sum_{j=1}^{n} \lambda_{j}\left(\left(x_{i j}^{a}\right)^{2}+\left(x_{i j}^{b}\right)^{2}\right) \leq \theta\left(\left(x_{i p}^{a}\right)^{2}+\left(x_{i p}^{b}\right)^{2}\right), \quad i=1, \ldots, m \\
& \sum_{j=1}^{n} \lambda_{j}\left(\left(y_{r j}^{a}\right)^{2}+\left(y_{r j}^{b}\right)^{2}\right) \geq\left(\left(y_{r p}^{a}\right)^{2}+\left(y_{r p}^{b}\right)^{2}\right), \quad r=1, \ldots, s \\
& \lambda_{j} \geq 0, \quad j=1, \ldots, n ; \quad \theta \text { free. }
\end{aligned}
$$


TABLE 1. Numerical example and the results of Esfandiari et al. [8] and modified model.

\begin{tabular}{lllllllllll}
\hline \hline DMU & $x_{1}^{a}$ & $x_{1}^{b}$ & $x_{2}^{a}$ & $x_{2}^{b}$ & $y_{1}^{a}$ & $y_{1}^{b}$ & $y_{2}^{a}$ & $y_{2}^{b}$ & $\begin{array}{l}\text { Esfandiari Modified } \\
\text { et al. }[8]\end{array}$ & \begin{tabular}{l} 
model \\
\hline 1
\end{tabular} \\
& 6 & 2 & 8 & 4 & 1 & 1 & 3 & 3 & 0.450 & 0.450 \\
2 & 7 & 8 & 3 & 5 & 8 & 6 & 5 & 7 & 1.000 & 1.000 \\
3 & 6 & 5 & 3 & 4 & 5 & 1 & 5 & 3 & 0.709 & 0.709 \\
4 & 9 & 5 & 6 & 6 & 5 & 3 & 3 & 4 & 0.362 & 0.362 \\
5 & 6 & 8 & 10 & 5 & 2 & 4 & 3 & 1 & 0.226 & 0.226 \\
6 & 3 & 7 & 5 & 3 & 3 & 6 & 7 & 3 & 1 & 1 \\
\hline
\end{tabular}

TABLE 2. Comparing the results of Esfandiari et al. [8] and modified model.

\begin{tabular}{lllllllllll}
\hline \hline DMU & $x_{1}^{a}$ & $x_{1}^{b}$ & $x_{2}^{a}$ & $x_{2}^{b}$ & $y_{1}^{a}$ & $y_{1}^{b}$ & $y_{2}^{a}$ & $y_{2}^{b}$ & $\begin{array}{l}\text { Esfandiari } \\
\text { et al. }[8]\end{array}$ & $\begin{array}{l}\text { Modified } \\
\text { model }\end{array}$ \\
\hline 1 & 6 & 2 & 8 & 4 & 1 & 1 & 3 & 3 & 0.450 & 0.450 \\
2 & 7 & 8 & 3 & 5 & 8 & 6 & 5 & 7 & 1.000 & 1.000 \\
3 & 6 & 5 & 0 & 5 & 5 & 1 & 5 & 3 & 0.648 & 0.709 \\
4 & 9 & 5 & 6 & 6 & 5 & 3 & 3 & 4 & 0.362 & 0.362 \\
5 & 6 & 8 & 2 & 11 & 2 & 4 & 3 & 1 & 0.226 & 0.226 \\
6 & 3 & 7 & 5 & 3 & 3 & 6 & 7 & 3 & 1.000 & 1.000 \\
\hline
\end{tabular}

Model (3.2) is a LP model to assess the efficiency of DMUs with complex data. In fact, with this approach, we converted a CCR model with complex numbers into a ordinary CCR model. Our model has all of the properties of Esfandiari et al. [8] model except in one case we are referring to changing the real and imaginary parts individually or simultaneously, its effect on unit performance should be observed. When we simultaneously change the real and imaginary parts of a complex number (one increases and the other decreases) so that the sum of the squares of these two numbers remains constant, in some cases, the model of Esfandiari et al. [8] changes the efficiency of DMUs but our model does not change. In the following, we illustrate this case with a numerical example.

\subsubsection{Numerical example}

Consider six DMUs with two complex inputs and outputs as follows Table 1:

$x^{a}$ and $y^{a}$ are considered as the real parts and $x^{b}$ and $y^{b}$ are considered as the imaginary parts of the parameters. The data in the last two columns show the efficiency scores by using Esfandiari et al. [8] and modified model. As seen, the performance results of the two models are the same. We now simultaneously change the second input of $\mathrm{DMU}_{3}$ from $3+4 \mathrm{i}$ to $0+5 \mathrm{i}$ and also the second input of $\mathrm{DMU}_{5}$ from $10+5 \mathrm{i}$ to $2+11 \mathrm{i}$, which the size of $3+4 \mathrm{i}$ with the size of $0+5 \mathrm{i}$ and the size of $10+5 \mathrm{i}$ with the size of $2+11 \mathrm{i}$ are equal, then recalculate the efficiency of the units Table 2.

As seen in the tenth column, the efficiency score of $\mathrm{DMU}_{3}$ by using Esfandiari et al. [8] model has changed from 0.709 to 0.648 and the effect of the real and imaginary parts on the efficiency of this DMU is determined separately, while the efficiency score of this DMU by using modified model (3.2) (see the last column) has not changed. The efficiency score of $\mathrm{DMU}_{5}$ by using both models has not changed. So, in some cases, Esfandiari et al. [8] model also has the weakness of the modified model. 


\subsection{DEA with fuzzy complex numbers}

To develop the model, we first need a method to deal with complexity of the data. Since the method proposed by Esfandiari et al. [8] (i.e. the concept of the size of the linear combination of inputs and outputs) complicate the model structure, we propose a modified method that is derived from Esfandiari et al. [8] idea. Using these two methods (modified method and the $\alpha$-level method), the CCR model with fuzzy complex data is transformed into a LP model with real data. Consider $n$ DMUs that each $\operatorname{DMU}_{j}(j=1, \ldots, n)$ products $s$ fuzzy complex outputs $\left(y_{r j}^{(l) a}, y_{r j}^{(m) a}, y_{r j}^{(u) a}\right)+\mathrm{i}\left(y_{r j}^{(l) b}, y_{r j}^{(m) b}, y_{r j}^{(u) b}\right)(r=1, \ldots, s)$ by consuming $m$ fuzzy complex inputs $\left(x_{i j}^{(l) a}, x_{i j}^{(m) a}, x_{i j}^{(u) a}\right)+\mathrm{i}\left(x_{i j}^{(l) b}, x_{i j}^{(m) b}, x_{i j}^{(u) b}\right)(i=1, \ldots, m)$. The both parts $\left(x_{i j}^{(l) a}, x_{i j}^{(m) a}, x_{i j}^{(u) a}\right)$ and $\left(x_{i j}^{(l) b}, x_{i j}^{(m) b}, x_{i j}^{(u) b}\right)$ denote, respectively, the real and imaginary parts of $i$ th complex input of $j$ th DMU $(j=1, \ldots, n)$, which the nature of both parts is stated as triangular fuzzy numbers. $(l),(m)$ and $(u)$ are, respectively, the lower, middle, and upper bounds of each part. The output parameters structure is also defined as the input parameters structure. All data are non-negative and there is at least an input and output greater than zero for each DMU. The CCR multiplier model (model (2.1)) in the fuzzy complex environment is shown as follows:

$$
\begin{aligned}
& \operatorname{Max} z=\sum_{r=1}^{s} u_{r}\left(\left(y_{r p}^{(l) a}, y_{r p}^{(m) a}, y_{r p}^{(u) a}\right)+\mathrm{i}\left(y_{r p}^{(l) b}, y_{r p}^{(m) b}, y_{r p}^{(u) b}\right)\right) \\
& \text { s.t. } \sum_{i=1}^{m} v_{i}\left(\left(x_{i p}^{(l) a}, x_{i p}^{(m) a}, x_{i p}^{(u) a}\right)+\mathrm{i}\left(x_{i p}^{(l) b}, x_{i p}^{(m) b}, x_{i p}^{(u) b}\right)\right)=1 \text {, } \\
& \sum_{r=1}^{s} u_{r}\left(\left(y_{r j}^{(l) a}, y_{r j}^{(m) a}, y_{r j}^{(u) a}\right)+\mathrm{i}\left(y_{r j}^{(l) b}, y_{r j}^{(m) b}, y_{r j}^{(u) b}\right)\right) \\
& -\sum_{i=1}^{m} v_{i}\left(\left(x_{i j}^{(l) a}, x_{i j}^{(m) a}, x_{i j}^{(u) a}\right)+\mathrm{i}\left(x_{i j}^{(l) b}, x_{i j}^{(m) b}, x_{i j}^{(u) b}\right)\right) \leq 0, \quad \forall j \\
& u_{r}, \quad v_{i} \geq 0 \text {, }
\end{aligned}
$$

Model (3.3) is a problem with fuzzy complex coefficients that incorporate the properties of the complexity and fuzziness. As mentioned, the main idea of this research is to present a new DEA model by using our proposed modified method and the $\alpha$-level approach proposed by Saati et al. [16]. To use the $\alpha$-level method, it first needs to convert complex coefficients of model (3.3) into real coefficients. For this purpose, we use the proposed modified method presented in the previous section and consider the size of FCNs. By applying this concept for model (3.3), model (3.4) is obtained:

$$
\begin{array}{lll}
\operatorname{Max} z=\sum_{r=1}^{s} u_{r}\left|\left(y_{r p}^{(l) a}, y_{r p}^{(m) a}, y_{r p}^{(u) a}\right)+\mathrm{i}\left(y_{r p}^{(l) b}, y_{r p}^{(m) b}, y_{r p}^{(u) b}\right)\right| & \\
\text { s.t. } & \sum_{i=1}^{m} v_{i}\left|\left(x_{i p}^{(l) a}, x_{i p}^{(m) a}, x_{i p}^{(u) a}\right)+\mathrm{i}\left(x_{i p}^{(l) b}, x_{i p}^{(m) b}, x_{i p}^{(u) b}\right)\right|=1, & \\
& \sum_{r=1}^{s} u_{r}\left|\left(y_{r j}^{(l) a}, y_{r j}^{(m) a}, y_{r j}^{(u) a}\right)+\mathrm{i}\left(y_{r j}^{(l) b}, y_{r j}^{(m) b}, y_{r j}^{(u) b}\right)\right| \\
& -\sum_{i=1}^{m} v_{i}\left|\left(x_{i j}^{(l) a}, x_{i j}^{(m) a}, x_{i j}^{(u) a}\right)+\mathrm{i}\left(x_{i j}^{(l) b}, x_{i j}^{(m) b}, x_{i j}^{(u) b}\right)\right| \leq 0, & \forall j . \\
& u_{r}, \quad v_{i} \geq 0, & \forall r, i
\end{array}
$$

Suppose $z_{1}=\left(a_{1}^{l}, a_{1}^{m}, a_{1}^{u}\right)$ and $z_{2}=\left(a_{2}^{l}, a_{2}^{m}, a_{2}^{u}\right)$ are two FNs and $z=\left(a^{l}, a^{m}, a^{u}\right)+\mathrm{i}\left(b^{l}, b^{m}, b^{u}\right)$ is a FCN, then the following relations are true $[5,20]$ :

$$
\begin{aligned}
z_{1}+z_{2} & =\left(a_{1}^{l}+a_{2}^{l}, a_{1}^{m}+a_{2}^{m}, a_{1}^{u}+a_{2}^{u}\right) \\
|z| & =\sqrt{\left(a^{l}, a^{m}, a^{u}\right)^{2}+\left(b^{l}, b^{m}, b^{u}\right)^{2}}=\sqrt{\left(\left(a^{l}\right)^{2}+\left(b^{l}\right)^{2},\left(a^{m}\right)^{2}+\left(b^{m}\right),\left(a^{u}\right)^{2}+\left(b^{u}\right)^{2}\right)} .
\end{aligned}
$$


The sign $\|$ shows the size of an FCN. Here, the second power of the size of FCNs is used for simplicity in computations. By applying the above relations, model (3.4) is converted into the following model:

$$
\begin{array}{lll}
\operatorname{Max} & z=\sum_{r=1}^{s} u_{r}\left(\left(y_{r p}^{(l) a}\right)^{2}+\left(y_{r p}^{(l) b}\right)^{2},\left(y_{r p}^{(m) a}\right)^{2}+\left(y_{r p}^{(m) b}\right)^{2},\left(y_{r p}^{(u) a}\right)^{2}+\left(y_{r p}^{(u) b}\right)^{2}\right) \\
\text { s.t. } \quad \sum_{i=1}^{m} v_{i}\left(\left(x_{i p}^{(l) a}\right)^{2}+\left(x_{i p}^{(l) b}\right)^{2},\left(x_{i p}^{(m) a}\right)^{2}+\left(x_{i p}^{(m) b}\right)^{2},\left(x_{i p}^{(u) a}\right)^{2}+\left(x_{i p}^{(u) b}\right)^{2}\right)=1, & \\
& \sum_{r=1}^{s} u_{r}\left(\left(y_{r j}^{(l) a}\right)^{2}+\left(y_{r j}^{(l) b}\right)^{2},\left(y_{r j}^{(m) a}\right)^{2}+\left(y_{r j}^{(m) b}\right)^{2},\left(y_{r j}^{(u) a}\right)^{2}+\left(y_{r j}^{(u) b}\right)^{2}\right) \\
\quad-\sum_{i=1}^{m} v_{i}\left(\left(x_{i j}^{(l) a}\right)^{2}+\left(x_{i j}^{(l) b}\right)^{2},\left(x_{i j}^{(m) a}\right)^{2}+\left(x_{i j}^{(m) b}\right)^{2},\left(x_{i j}^{(u) a}\right)^{2}+\left(x_{i j}^{(u) b}\right)^{2}\right) \leq 0, & \forall j \\
u_{r}, \quad v_{i} \geq 0 & \forall r, i
\end{array}
$$

The above LP model is a DEA model with triangular fuzzy data in which $\left(y_{r j}^{(l) a}\right)^{2}+\left(y_{r j}^{(l) b}\right)^{2},\left(y_{r j}^{(m) a}\right)^{2}+\left(y_{r j}^{(m) b}\right)^{2}$ and $\left(y_{r j}^{(u) a}\right)^{2}+\left(y_{r j}^{(u) b}\right)^{2}$ are the real values relation to the lower, middle and upper bounds of $r$ th output, respectively, and $\left(x_{i j}^{(l) a}\right)^{2}+\left(x_{i j}^{(l) b}\right)^{2},\left(x_{i j}^{(m) a}\right)^{2}+\left(x_{i j}^{(m) b}\right)^{2}$ and $\left(x_{i j}^{(u) a}\right)^{2}+\left(x_{i j}^{(u) b}\right)^{2}$ indicate the real values relation to the lower, middle and upper bounds of $i$ th input of $j$ th $\operatorname{DMU}(j=1, \ldots, n)$, respectively. Now we can apply the concept of the $\alpha$-cut for model (3.5) to deal with fuzziness of the data. By applying the concept of $\alpha$-cut proposed by Saati et al. [16] for the above model, the final model (3.6) is obtained:

$$
\begin{aligned}
& \operatorname{Max} z=\sum_{r=1}^{s} \bar{y}_{r p} \\
& \text { s.t. } \sum_{i=1}^{m} \bar{x}_{i p}=1 \text {, } \\
& \sum_{r=1}^{s} \bar{y}_{r j}-\sum_{i=1}^{m} \bar{x}_{i j} \leq 0 \\
& v_{i}\left(\alpha\left(\left(x_{i j}^{(m) a}\right)^{2}+\left(x_{i j}^{(m) b}\right)^{2}\right)+(1-\alpha)\left(\left(x_{i j}^{(l) a}\right)^{2}+\left(x_{i j}^{(l) b}\right)^{2}\right)\right) \leq \bar{x}_{i j} \\
& \leq v_{i}\left(\alpha\left(\left(x_{i j}^{(m) a}\right)^{2}+\left(x_{i j}^{(m) b}\right)^{2}\right)+(1-\alpha)\left(\left(x_{i j}^{(u) a}\right)^{2}+\left(x_{i j}^{(u) b}\right)^{2}\right)\right), \quad \forall i, j \\
& u_{i}\left(\alpha\left(\left(y_{r j}^{(m) a}\right)^{2}+\left(y_{r j}^{(m) b}\right)^{2}\right)+(1-\alpha)\left(\left(y_{r j}^{(l) a}\right)^{2}+\left(y_{r j}^{(l) b}\right)^{2}\right)\right) \leq \bar{y}_{r j} \\
& \leq u_{i}\left(\alpha\left(\left(y_{r j}^{(m) a}\right)^{2}+\left(y_{r j}^{(m) b}\right)^{2}\right)+(1-\alpha)\left(\left(y_{r j}^{(u) a}\right)^{2}+\left(y_{r j}^{(u) b}\right)^{2}\right)\right), \quad \forall r, j \\
& u_{r}, v_{i} \geq 0 \text {, }
\end{aligned}
$$

The above model is a parametric LP model to evaluate the efficiency of DMUs with FCNs that simultaneously deals with complexity and fuzziness of the data. The objective function of the proposed model like the objective function of CCR model yields the efficiency score of the DMU under consideration. $\alpha \in(0,1]$ is a parameter and for any value of $\alpha$ in model (3.6) we can obtain an optimal solution; therefore, we can have a list of optimal solutions for the different values of $\alpha$.

\subsection{Ranking model}

The standard DEA models always calculate the efficiency score of DMUs less than or equal to one. The inefficient DMUs are ranked with their inefficiency values, but the efficient DMUs that all have the efficiency value equal to 1 have no ranking can be obtained. Andersen and Petersen [1] developed a ranking model by DEA approach to rank the efficient DMUs. In another study, Saati et al. [16] presented a ranking model for fuzzy DEA. Suppose that some DMUs in the fuzzy complex environment evaluated by model (3.6) become efficient. So, it is need to rank these DMUs. There is no ranking model with DEA approach that can rank DMUs with FCN. In this section, we present a new ranking model. Model (2.2) by considering the fuzzy complex inputs and outputs as 
$\left(x_{i j}^{(l) a}, x_{i j}^{(m) a}, x_{i j}^{(u) a}\right)+\mathrm{i}\left(x_{i j}^{(l) b}, x_{i j}^{(m) b}, x_{i j}^{(u) b}\right)(i=1, \ldots, m)$ and $\left(y_{r j}^{(l) a}, y_{r j}^{(m) a}, y_{r j}^{(u) a}\right)+\mathrm{i}\left(y_{r j}^{(l) b}, y_{r j}^{(m) b}, y_{r j}^{(u) b}\right)(r=1, \ldots, s)$ is demonstrated as following:

$$
\begin{array}{lll}
\text { Min } z=\theta & & \\
\text { s.t. } & \sum_{j=1}^{n} \lambda_{j}\left(\left(x_{i j}^{(l) a}, x_{i j}^{(m) a}, x_{i j}^{(u) a}\right)+\mathrm{i}\left(x_{i j}^{(l) b}, x_{i j}^{(m) b}, x_{i j}^{(u) b}\right)\right) & \\
\quad \leq \theta\left(\left(x_{i p}^{(l) a}, x_{i p}^{(m) a}, x_{i p}^{(u) a}\right)+\mathrm{i}\left(x_{i p}^{(l) b}, x_{i p}^{(m) b}, x_{i p}^{(u) b}\right)\right), & \forall i \\
& \sum_{j=1}^{n} \lambda_{j}\left(\left(y_{r j}^{(l) a}, y_{r j}^{(m) a}, y_{r j}^{(u) a}\right)+\mathrm{i}\left(y_{r j}^{(l) b}, y_{r j}^{(m) b}, y_{r j}^{(u) b}\right)\right) & \\
\quad \geq\left(y_{r p}^{(l) a} y_{r p}^{(m) a}, y_{r p}^{(u) a}\right)+\mathrm{i}\left(y_{r p}^{(l) b}, y_{r p}^{(m) b}, y_{r p}^{(u) b}\right), & \forall r \\
\quad \lambda_{j} \geq 0, & \forall j .
\end{array}
$$

Similar to the process of converting model (3.3) to model (3.6), model (3.7) is developed to model (3.8):

$$
\begin{array}{ll}
\text { Min } z=\theta & \\
\text { s.t. } \sum_{j=1}^{n} \lambda_{j}\left(\alpha\left(\left(x_{i j}^{(m) a}\right)^{2}+\left(x_{i j}^{(m) b}\right)^{2}\right)+(1-\alpha)\left(\left(x_{i j}^{(u) a}\right)^{2}+\left(x_{i j}^{(u) b}\right)^{2}\right)\right) & \\
\quad \leq \theta\left(\alpha\left(\left(x_{i p}^{(m) a}\right)^{2}+\left(x_{i p}^{(m) b}\right)^{2}\right)+(1-\alpha)\left(\left(x_{i p}^{(l) a}\right)^{2}+\left(x_{i p}^{(l) b}\right)^{2}\right)\right), & \forall i \\
\sum_{j=1}^{n} \lambda_{j}\left(\alpha\left(\left(x_{i j}^{(m) a}\right)^{2}+\left(x_{i j}^{(m) b}\right)^{2}\right)+(1-\alpha)\left(\left(y_{r j}^{(l) a}\right)^{2}+\left(y_{r j}^{(l) b}\right)^{2}\right)\right) & \\
\quad \geq \alpha\left(\left(y_{r p}^{(m) a}\right)^{2}+\left(y_{r p}^{(m) b}\right)^{2}\right)+(1-\alpha)\left(\left(y_{r p}^{(u) a}\right)^{2}+\left(x_{r p}^{(u) b}\right)^{2}\right), & \forall r \\
\lambda_{j} \geq 0 & \forall j .
\end{array}
$$

Model (3.8) is a parametric LP problem in which $\alpha \in(0,1]$ is a parameter. It is noted that for each of the value $\alpha$, there is an optimal solution. Thus, we can achieve the different optimal solutions with the different values of $\alpha$.

\section{EFFiCIENCY MEASUREMENT AT THE GENERATORS OF THE STEAM POWER PLANTS}

The case study used in this research is the generators of the steam power plants. The role of a power plant is to efficiently transform fuel into electricity (electric power). The power plants have different types depending on their fuel consumption which among them the steam power plants produce the most electricity (about $86 \%$ of all electricity generation) in the worldwide. The power generation process at the power plant can be divided into two stages where the first stage (including the boilers and turbines) converts fuel into the mechanical power and the second stage (including the generators) converts the mechanical power into the electric power. The most effective part of a steam generator is the generator, which consist of two main parts of rotating and static. Mechanical power with its kinetic energy rotates the generator which by inducing the excitation voltage and the excitation current in the rotating section, a rotating magnetic field is formed and finally the electricity is stored in the stator section. The nature of the input data of the generators are fuzzy and the output data of the generators have the fuzzy complex nature. The electric power as the output data is divided to two parts of the active power and the reactive power in which the active and reactive powers are called the real and imaginary parts of a complex number, respectively and the data of both powers are available as the triangular fuzzy. According to their undeniable role of the generators in power generation, we apply the proposed model on the real data of the generators of the steam power plants (as DMUs). We provided the average data of the steam power plants generators for the year 2015. This data was collected from Tavanir Company. See data in Table 3:

The excitation current $\left(x_{1}\right)$, excitation voltage $\left(x_{2}\right)$, and mechanical energy $\left(x_{3}\right)$ are the inputs data of the generators whose the real part is fuzzy and their imaginary part is zero. 


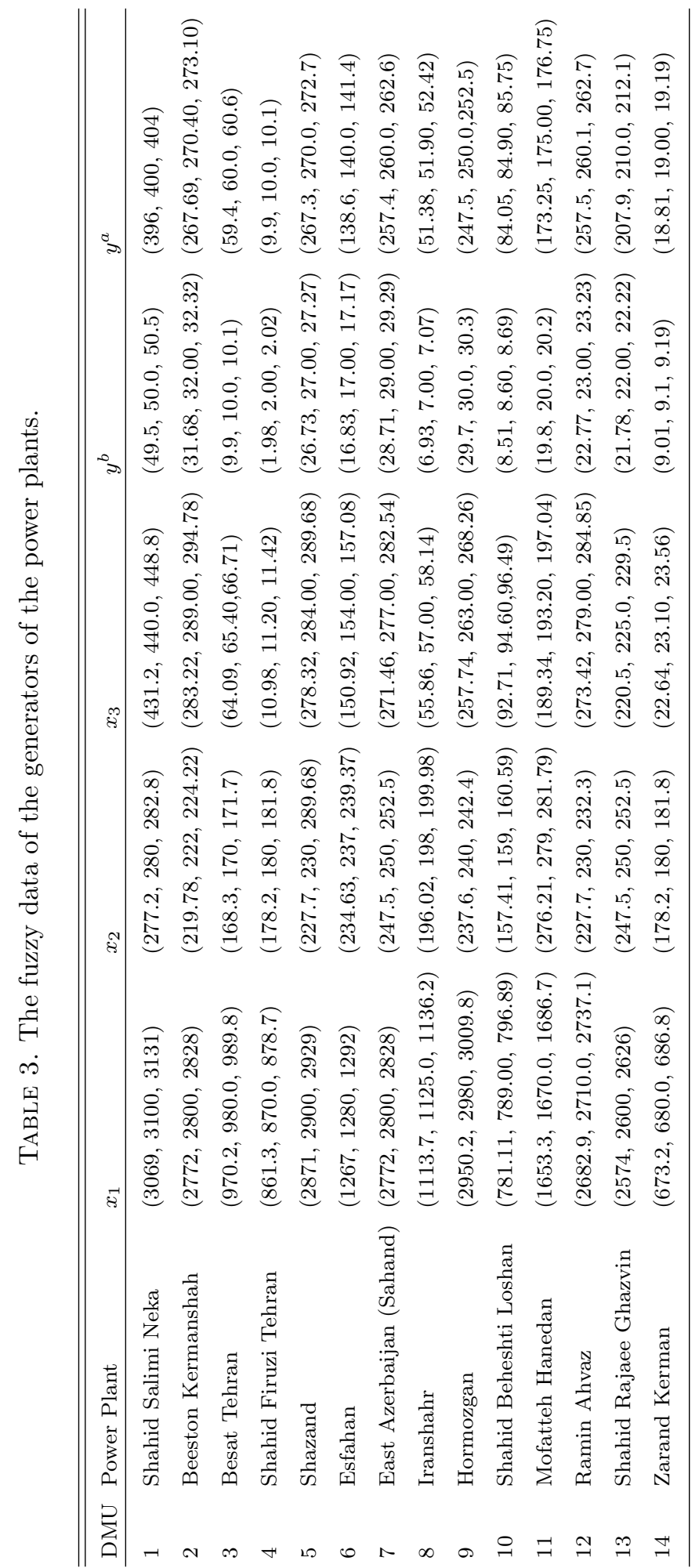




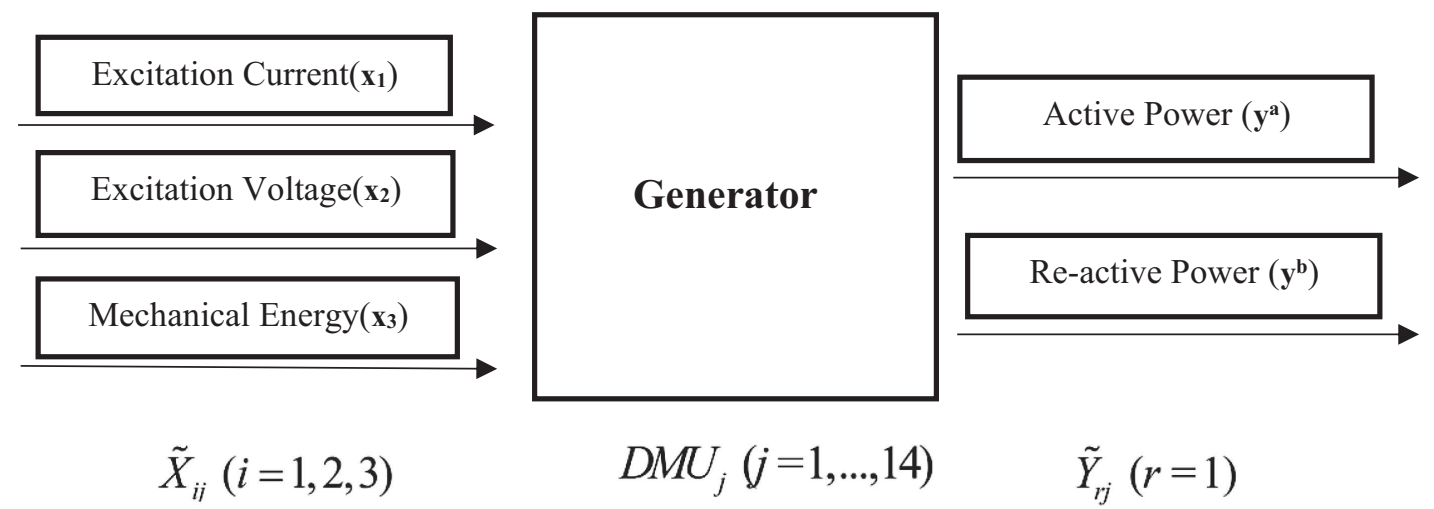

FiguRE 1. Schematic view of a generator as a DMU.

The electric power $(\tilde{y})$ is the only the fuzzy complex output of the generators is sent to the consumption network and consists of two parts of the active power $\left(\tilde{y}^{a}\right)$ and the reactive power $\left(\tilde{y}^{b}\right)$ whose fuzzy values were listed in the last two columns of Table 3. Figure 1 show the structure of the inputs and outputs of a generator in a steam power plant as a DMU.

The excitation current and voltage $\left(x_{1}, x_{2}\right)$. The rotor section of the generator is induced by using these two the inputs to create a magnetic field.

Mechanical power $\left(x_{3}\right)$. In the first stage, the steam generated by burning of the fuel by affecting into the turbine blades rotates them and is generated mechanical power. This power enters the generator as input.

Electric power $(\boldsymbol{y})$. This power has a complex nature is produced in the stator section of the generator and is emitted by the generator.

Active power $\left(\boldsymbol{y}_{r j}^{a}\right)$. It is a part of the electric power that its performance can be seen. For instance, it turns on the lamps. It is measured by megawatt.

Reactive power $\left(\boldsymbol{y}_{\boldsymbol{r} j}^{\boldsymbol{b}}\right)$. This power as the imaginary part of the electric power along with the active power is transmitted from the generator into the consumer networks and by managing the voltage level, transmits the active power through the lines. We cannot see the performance of this power with the eye (e.g. does not light the lamp). Its unit of measurement is Mega-var.

It should be noted that the fuzzy data in Table 3 are asymmetric triangular.

We run the proposed models (3.6) and (3.8) in the GAMS software for the generators data of 14 steam power plants. Results of implementation of these two models yielded the efficiency values of 14 DMUs and their ranking with different $\alpha$ values. Results were listed in Table 4.

As seen in Table 4, the efficiency scores of DMUs, which calculated by model (3.6) were listed in the first section, and the second section of the table shows the rank DMUs by model (3.8). The third section of Table 4 (last column) demonstrates the efficiency values by the modified model (3.2) considering the mean limit of data of the generators.

Consider $\alpha=0.25$ in the fourth column, the efficiency score of all DMUs except $\mathrm{DMU}_{4}$ and $\mathrm{DMU}_{14}$ is equal to unity. In the fifth and sixth columns, the numbers of inefficient DMUs by $\alpha=0.5$ and $\alpha=0.75$, respectively is four and eight. It is observed the numbers of the inefficient DMUs increases by increasing $\alpha$ value $\mathrm{DMU}_{4}$ and DMU14 have the greatest number of inefficiency (in four case) by different $\alpha$ values. While DMUs 1, 5, 9 become efficient for all $\alpha \in(0,1]$, in other word, they are not sensitive to alpha changes and maintained their efficiency. The seventh column show the efficiency scores of DMUs by $\alpha=1$, which in this case the proposed model (3.6) calculated the efficiency values by mean limit of data such as the multiplier model of CCR with complex data. 
The modified model in (3.2) is dual of the multiplier model of CCR with complex data, so as seen the results of the proposed model (3.6) by $\alpha=1$ is equal to the results of the modified model in (3.2) with the mean limit of data (see the last column). The efficient DMUs with different $\alpha$ values per column cannot be ranked and the ranking model (3.8) was used to rank them. By implementing this model, the efficiency score of DMUs were already efficient become greater than unity and the efficiency of the inefficient DMUs does not changed. For an example consider the results of model (3.6) by $\alpha=0.75$ in the sixth column in which DMUs $1,2,5,7,9$ and 12 are efficient. The ranking results of these efficient DMUs by implementing model (3.8) were presented in the column related to $\alpha=0.75$ in the second part of Table 4 which the their ranking order from large to small is as DMUs 9, 5, 1, 2 and 7. By $\alpha=1$, the results of model (3.6) are exactly the same as the results of model (3.8). Because model (3.6) considering $\alpha=1$ is equivalent to the multiplier model of DEA with complex data and the model (3.8) by $\alpha=1$ is equivalent to the envelopment model of DEA with complex data presented in (3.2) which these two envelopment and multiplier models are the primal and dual models of each other with the same optimal solution values. See at the second part of Table 4, the most ranking of the efficiencies by all $\alpha$ values is associated with $\mathrm{DMU}_{1}$ (Shahid Salimi Power Plant in Neka), and the lowest ranking is for $\mathrm{DMU}_{4}$ (Shahid Firouzi Power Plant in the Tarasht section of Tehran). These results were somewhat predictable, because the technology type or design of the generators is one of the influential factors in the efficiency value of the generators in power plants. Shahid Salimi Power Plant $\left(\mathrm{DMU}_{1}\right)$ as one of the country's most important assets and largest electricity producer in Iran, has high technology in internal equipment, and Shahid Firouzi Power Plant $\left(\mathrm{DMU}_{4}\right)$ is one of the oldest steam power plants who's the steam units were operated in the late 1950s.

\section{Conclusions}

To calculate the performance of industries whose input and output data are fuzzy complex, none of the existing DEA models can be used to simultaneously deal with complexity and fuzziness. In this paper, we introduced a new DEA model with FCNs to evaluate the efficiency of these industries and then developed a ranking model to rank the efficient DMUs. The input-oriented multiplier model of CCR was extended for FCNs by using the data size-based method and the $\alpha$-cut based approach. We initially got the idea from Esfandiari et al. [8] method and presented a simpler model than their model named the modified model. For meet with the complexity of data, we used the proposed modified method (i.e. the size data) and transformed the multiplier model of CCR with FCNs into the CCR model with the FNs, and then used the $\alpha$-cut based approach presented by Saati et al. [16] for dealing with the fuzziness of data. Finally, by applying these two methods, the CCR model with FCNs was converted into the CCR model with the real data. In the following, we extended the input-oriented envelopment model of CCR for FCNs to introduce the ranking model. The ranking model was also developed by using the two methods mentioned above.

A case study in the generators of the steam power plants was presented to demonstrate the applicability of the proposed models in the power industry. The data set from 14 generators of the steam power-plants in Iran with three real fuzzy inputs (excitation current, excitation voltage, mechanical energy) and a fuzzy complex output (reactive power) was considered in which the fuzzy parts of data are asymmetric triangular. The results of the running models with different $\alpha$ values for this case study in the GAMS software were assessed. The results showed by increasing $\alpha$ the efficiency scores of the generators of the power plants by new DEA model and their ranking model became gradually closer to the results of the CCR model with complex data (results of the modified model in the last column) until the results of these two models (model (3.6) and model (3.8)) with the results of the modified model (model (3.2) with the mean limit of data) become equal in $\alpha=1$. We introduced a new DEA model for FCNs and then developed a ranking model for it. The results of applying the proposed models in the case study were satisfactory and the calculations were done simple. Because this idea was proposed for the first time and there was no article relation to it, hence we compared the results of the proposed model with the experimental results. The proposed approach of this research can be used for the 
existing DEA models such as network, dynamic, Malmquist etc. in which the inputs and outputs data of DMUs are FCNs.

Acknowledgements. The authors would like to thank the Iran Electricity Management, Distribution, Transfer, and Production (Tavanir Company) for providing the data and their guidance.

\section{REFERENCES}

[1] P. Andersen and N.C. Andersen, A procedure for ranking efficient units in data envelopment analysis. Manage. Sci. 39 (1993) 1261-1264.

[2] M. Azadi, M. Jafarian, R. Farzipoor Saen and S.M. Mirhedayatian, A new fuzzy DEA model for evaluation of efficiency and effectiveness of suppliers in sustainable supply chain management context. Comput. Oper. Res. 54 (2015) $274-285$.

[3] X. Bai, F. Zhang2 and Y. Liu, Modeling Fuzzy Data Envelopment Analysis under robust input and output data. RAIRO:OR 52 (2018) 619-643.

[4] J.J. Buckley, Fuzzy complex numbers. Fuzzy Sets Syst. 33 (1989) 333-345.

[5] D. Chakraborty and D. Guha, Addition of two generalized fuzzy numbers. Int. J. Ind. Math. 2 (2010) 9-20.

[6] A. Charnes, W.W. Cooper and E. Rhodes, Measuring the efficiency of decision-making units. Eur. J. Oper. Res. 2 (1978) 429-44.

[7] R.V. Churchill, J.W. Brown and R.F. v, Complex Variables and Applications. McGraw-Hill, New York, NY (1974).

[8] M. Esfandiari, S. Saati, M. Navabakhsh and K. Khalili-Damghani, A novel data envelopment analysis model with complex numbers: measuring the efficiency of electric generators in steam power plants. Oper. Res. Decis. 29 (2019) 41-52.

[9] M.J. Farrell, The measurement of productive efficiency. J. R. Stat. Soc. Ser. A 120 (1957) 253-281.

[10] P. Guo and H. Tanaka, Fuzzy DEA: a perceptual evaluation method. Fuzzy Sets Syst. 119 (2001) 149-160.

[11] P. Guo, H. Tanaka and M. Inuiguchi, Self-organizing fuzzy aggregation models to rank the objects with multiple attributes. IEEE Trans. Syst. Man Cybern. Part A Syst. Humans 30 (2000) 573-580.

[12] A. Hatami-Marbini, A. Emrouznejad and M. Tavana, A taxonomy and review of the fuzzy data envelopment analysis literature: two decades in the making. Eur. J. Oper. Res. 214 (2011) 457-472.

[13] S. Lertworasirikul, S.C. Fang, J.A. Joines and H.L.W. Nuttle, A possibility approach to fuzzy data envelopment analysis. In: Vol. 6 of Proceedings of the Joint Conference On Information Sciences. Duke University/Association for Intelligent Machinery, Durham, NC (2002), 176-179.

[14] S. Lertworasirikul, S.C. Fang, H.L.W. Nuttle and J.A. Joines, Fuzzy data envelopment analysis. In: Proceedings of the 9th Bellman Continuum, Beijing (2002), 342.

[15] S.H. Mirzaei and A. Salehi, Ranking efficient DMUs using the Tchebycheff norm with fuzzy data in DEA. Int. J. Res. Ind. Eng. 8 (2019) 158-175.

[16] S. Saati, A. Memariani and G.R. Jahanshahloo, Efficiency analysis and ranking of DMUs with fuzzy data. Fuzzy Optim. Decis. Making 1 (2002) 255-267.

[17] J.K. Sengupta, A fuzzy system approach in Data Envelopment Analysis. Comput. Math. App. 24 (1992) $259-266$.

[18] M. Tavassoli, R. Farzipoor-Saen and D. Mohamadi Zanjirani, Assessing sustainability of suppliers: a novel stochastic-fuzzy DEA model. Sustainable Prod. Consump. 21 (2020) 78-91.

[19] H. Tlig and A. Hamed, Assessing the Efficiency of commercial Tunisian Banks using fuzzy Data Envelopment Analysis. J. Data Envelopment Anal. Decis. Sci. 2 (2017) 14-27.

[20] F. Xin and Sh. Qiang, Fuzzy complex numbers and their application for classifiers performance evaluation. Pattern Recognit. 44 (2011) 1403-1417. 